\title{
Morphological Conservation in Human-Animal Hybrids in Science Fiction and Fantasy Settings: Is Our Imagination as Free as We Think It Is?
}

\author{
Matthieu J. Guitton ${ }^{1,2}$ \\ ${ }^{1}$ Faculty of Medicine, Laval University, Quebec City, Canada \\ ${ }^{2}$ Institut Universitaire en Santé Mentale de Québec, Quebec City, Canada \\ Email: matthieu.guitton@fmed.ulaval.ca \\ Received March $1^{\text {st }}, 2013$; revised April $3^{\text {rd }}, 2013$; accepted May $20^{\text {th }}, 2013$
}

Copyright (C) 2013 Matthieu J. Guitton. This is an open access article distributed under the Creative Commons Attribution License, which permits unrestricted use, distribution, and reproduction in any medium, provided the original work is properly cited.

\begin{abstract}
The question of whether human imagination knows no boundaries or is, alternatively, constrained by conscious or unconscious cognitive templates is a key issue in defining human mind. We try here to address this extremely large question by focusing on one particular element of imaginary creations, the specific case of human-animal hybrids. Human-animal hybrids are common inhabitants of human imaginary spaces, being regularly encountered across numerous mythologies, as well as in modern popular culture. If human imagination was unconstrained, it would be expected that such hybrid creatures would display roughly half human and half animalistic features. Using several different popular science fiction and fantasy settings, we conducted an analysis of the morphological traits of human-animal hybrids, both anatomical and phenotypic. Surprisingly, we observed extremely high conservation of human morphological traits in human-animal hybrids, with a contrasting high use of phenotypic ("cosmetic") alterations, and with highly stereotyped patterns of morphological alterations. While these alterations were independent of the setting considered, shape alterations were setting-dependent and used as a way to increase internal coherence. Finally, important gender differences were observed, as female human-animal hybrids retained significantly more human traits than males did, suggesting that conservation of female appearance may bear essential evolutionary importance. Taken together, these results demonstrate the existence of strong cognitive templates which frame and limit the expression of the capacity of human imagination, and unveil some of the psychological mechanisms which constrain the emergence of imaginary spaces.
\end{abstract}

Keywords: Anthropomorphism; Gender Differences; Human Imagination; Imaginary Settings; Science Fiction and Fantasy; Zoomorphism

\section{Introduction}

Human beings take great pride in the capacity of their imagination. From mythologies and traditional folklores to modern science fiction and fantasy works, human dreams and fantasies seem to know neither limits nor boundaries. However, are we as free as we think we are when it comes to creating new universes, beings, or creatures; or, alternatively, is imagination constrained by-conscious or unconscious - cognitive templates? Do coherent imaginary spaces emerge only from random imaginary creation process, or from some hidden forms of cognitive determinants such as general psychological constraints of the human mind?

Addressing directly this extremely large question would however be an impossible challenge. By definition, coherent imaginary universes encompass numerous components, and too many factors could be involved in their creation. A good way to approach this fundamental question is to analyze the way modern fictional settings are built. Indeed, while mythological systems and traditional folkloric tales are by definition highly culturally constrained, modern science fiction and fantasy settings are arguably more "free", in the sense that their creators are supposed to be allowed to actively express their creativity to transcend, and often transgress, the limits of reality. Visual proximity to standard human appearance has been shown to be critical in virtual spaces (Lortie \& Guitton, 2011). Therefore, we choose one particular element of imaginary universes - the specific case of human-animal hybrids - and investigated it quantitatively in order to unveil the putative existence of cognitive mechanisms underlying their genesis.

Human-animal hybrids (in the context of this study, "human-animal hybrids" will be considered as isomorphic to anthropotheriomorphic creatures, i.e., beings displaying both human and animalistic features) are common inhabitants of human imaginary spaces. Such creatures are regularly encountered across numerous mythologies (Eliade, 1965; Black \& Green, 1992; Pinch, 2004), as well as in modern popular culture. From the Hawkmen of the planet Mongo (from the comic strip Flash Gordon) to the Valusian Serpent Men of Howard's Thurian Age (Howard, 1929) or the Draconians, reptilian humanoid extraterrestrials from the British television series Doctor Who, fictional creatures featuring morphological traits both from humans and from Earth animals are a common element of 


\section{J. GUITTON}

various science fiction and fantasy settings. Such human-animal hybrids can be extremely various, since any possible living Earth creature could give rise to human-animal hybrids in one fictional setting or another. If human imagination was unconstrained, it would be expected that such hybrid creatures would display roughly half human and half animalistic features.

Using three different popular science fiction and fantasy settings taken from cinema and television, we conducted an analysis of the morphological-both anatomical and phenotypictraits of human-animal hybrids. Our results unveil surprisingly high conservation of human traits in human-animal hybrids, whatever the setting studied, alongside with extremely conserved anatomical patterns of alterations. In contrast, shape alterations appear to be setting-dependant. Finally, the results highlight significant gender differences, female human-animal hybrids retaining much more human traits than males do. Taken together, these results suggest the existence of constraining cognitive models of how living organisms should look, which are able to overpower the creative processes involved in imagination.

\section{Material and Methods}

\section{General Protocol}

Human-animal hybrids were systematically searched for in three different settings (see below). Regardless of the setting, only adult subjects were considered. For each human-animal hybrid identified, anatomical alterations were calculated as a percentage of the body which differed from a standard human body. To do so, we used body charts dividing the human body into proportional areas (Lomanowska \& Guitton, 2012). The Lund and Browder chart is a commonly used body chart which was developed to assess the amount of skin damage in burn victims (Harvey et al., 1984; Kasten et al., 2011). The original chart divides the body into a total of 33 areas. In order to increase its accuracy and resolution power, we further subdivided the largest areas (e.g., upper and lower legs, torso) and areas with characteristic features (hand subdivisions, spine), resulting in a total of 49 areas. The use of these charts has been demonstrated to have a high degree of reliability and consistency (Wachtel et al., 2000; Lomanowska \& Guitton, 2012).

For each subject, two measures were made for each area: presence or absence of morphological alterations (i.e., modification altering the skeletal structure of the subject), and presence or absence of phenotypic alterations (i.e., any phenotypic element not found in a human being, e.g., non-human skin color, fur, feathers, scales, etc.). The scores were then converted into two percentages: the percentage of the body morphologically altered, and the percentage of the body phenotypically altered.

For each subject, the putative presence of additional limbs (arms, legs, wings, or tails) was noted. Finally, global shape alterations (taller, smaller, fatter, or thinner than a normal human) were also recorded.

\section{Corpus 1: Star Wars}

We selected as the first model of a science fiction and fantasy setting the fictional universe of "Star Wars". Centered around two series of three films (the original trilogy released between 1977 and 1983; and the prequel trilogy released between 1999 and 2005), Star Wars is one of the most popular science fiction and fantasy universes, spanning from movies and novels to virtual spaces in which very large fan communities are extremely active (Guitton, 2012a, 2012b).

Star Wars features the classical elements of a science fiction setting: technologically advanced civilizations, numeroushumanoid and non-humanoid - alien species, spaceships and space travel between distant planets, futuristic weaponry, intelligent droids, etc. But interestingly, Star Wars also features the classical elements of fantasy: the prominent role of a mystical power referred to the "Force", the dichotomous opposition of a monastic order of mystically empowered "knights" (the "Jedi") and an evil group of equally empowered hidden warriors (the "Sith"), characters engaged in epic adventures and far-travelling quests. Lightsaber duels and Force powers provide a portrayal extremely similar to a classical "swords and sorcery" setting. Hence, Star Wars encompasses characteristics from both science fiction and fantasy, and represents a perfect example of what such a universe could be.

Since our aim was to characterize the capacity of human imagination in a manner that was as unbiased as possible, we focused our sampling on the movies of the prequel trilogy with Episode I: The Phantom Menace, released on May 19, 1999; Episode II: Attack of the Clones, released on May 16, 2002; and Episode III: Revenge of the Sith, released on May 19, 2005, to avoid as much as possible the limitations related to the special effects technologies of the early 80 s compared to the computer-assisted possibilities of the early 2000s. Among the numerous aliens of the movies, several displayed animal features, and were clearly originally designed as human-animal hybrids (Figure 1). Human-animal hybrids were sampled from the movies, and their anatomical and phenotypic characteristics were quantified as described above. For each species, only one individual for a given morphological type was counted. However, if several different morphological types existed within one species (for instance the Gungan, with a standard morphological type represented by the character of "Jar Jar Binks", and with a different morphological type seen with "Boss Rugor Nass"), all of them were counted. When the movies were not enough for evaluating the morphological (anatomical or phenotypic) characteristics of the subject, or in case of genderambiguous characters, complementary information was obtained from the extensive and authoritative online database of the Star Wars universe "Wookieepedia" (http://starwars.wikia. com/wiki/).
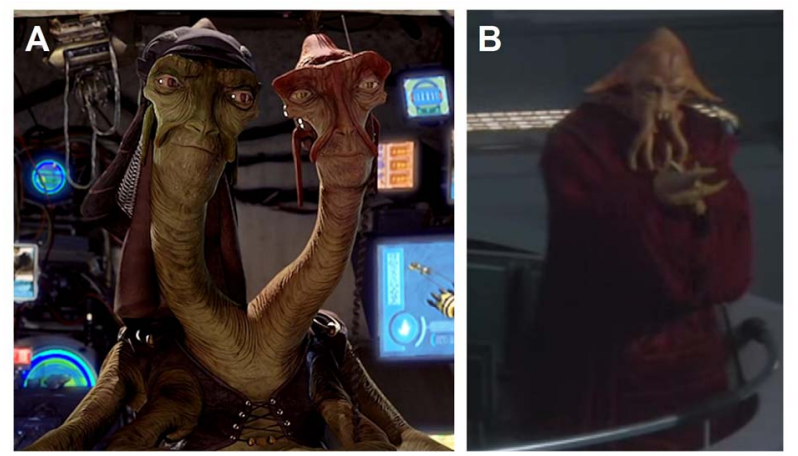

Figure 1.

Example of a human-animal hybrid in the Star Wars setting. (A) A two-headed alien that was not considered as a human-animal hybrid. (B) An alien (in this case a Quarren) that was considered as a hybrid between a human and a cephalopod. 


\section{Corpus 2: He-Man and the Masters of the Universe}

To overcome the limitations of the Star Wars universe setting as a model and to extend our results, we selected another science fiction and fantasy corpus: the setting of "He-Man and the Masters of the Universe". He-Man is an extremely large media franchise created by Mattel in 1981, which has been extended since then to include four animated series, numerous comic books, films, toy lines and other products, all of them relating to the adventures of Prince Adam, a member of the royal family of the fictional planet Eternia. Thanks to a mystically imbued sword, Prince Adam can become He-Man, a Conan-like barbaric warrior with super-human strength fighting with his allies, the Heroic Warriors, against evil forces lead by the infamous Skeletor. Like the Star Wars universe, the setting of He-Man and the Masters of the Universe has the characteristic of combining both elements of swords and sorcery, and of science fiction, featuring alongside heroic warriors and villainous foes, ancestral magic, powerful artifacts, mystical creatures, and science fiction technologies.

For the present study, we selected the most recent material available from this franchise, i.e., the "He-Man and the Masters of the Universe" animated television series developed between 2002 and 2004 by Mike Young Productions. All human-animal hybrids featured in the 39 episodes of the two seasons of the series were sampled and included in this study. The sampling approach was the same as described above: for a given species, only one individual per morphological type was selected. For instance, all the cat warriors of Season 1 Episode 10 "Dragon's Brood" were morphologically identical, hence they were counted only one time; in contrast, the Andreenids, a bee-human hybrid race, displayed three distinct morphologies for the males: the one of the heroic warrior Buzz-Off, the bee soldiers who were taller and displayed different chitinous alterations, and the fatter and smaller basic bee workers, which, in addition to the female Andreenid bee queen, resulted in four different morphological types, hence four counts in the selected corpus.

Excluded from the sampling were hybrids of human and mythical creatures (for instance, "King Hiss", a hybrid of a human and a hydra, leader of the Snake Men and main villain of Season 2, first seen extremely briefly in Season 1 Episode 21 "Snake Pit", then in most of the episodes of Season 2; or "Snake Face", a hybrid of a human and a gorgon, first seen in Season 2 Episode 4 "Rise of the Snake Men, Part 1", Figure 2). Excluded were also characters under transient transformation resulting in a hybrid of a human and a hybrid, or between two hybrids (mainly seen in Season 2 Episode 8 "Second Skin", with a spell hybridizing humanoids with snake men, resulting in human-lion/human-snake hybrids (cat warriors under the spell) or human/human-snake hybrids (the heroic warriors Man-at-Arms, Teela and Mekaneck under the spell). Indeed, in the case of such complex hybridization situations, the theoretical 50/50 repartition of human and animal features could not be assumed, and the results would have biased our sampling.

Two characters that evolved significantly during the series (namely Stinkor (from a human-sloth hybrid to a human-skunk hybrid, and Webstor, with two independent and strongly different forms of human-spider hybrid) were thus counted twice, once for each human-animal hybrid form.

\section{Corpus 3: She-Ra: Princess of Power}

Since both the Star Wars movies and the two seasons of the
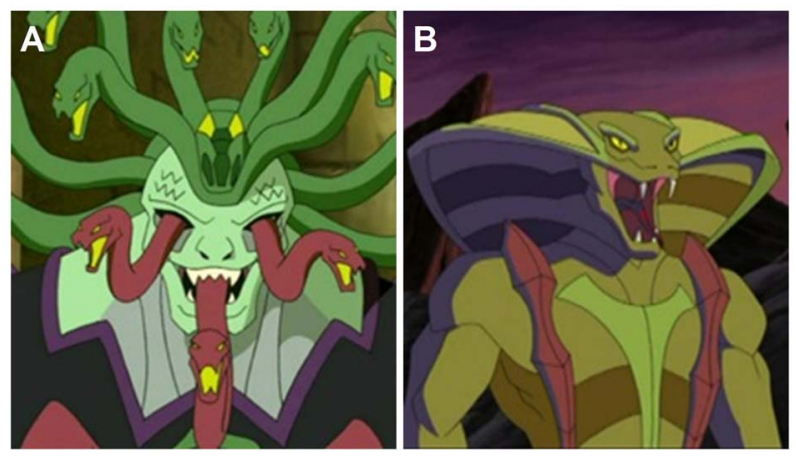

Figure 2.

Example of a human-animal hybrid in the New He-Man setting. Two Snake Men, (A) Snake Face, a hybrid of a human and a mythological creature (a gorgon), which was not included in our sampling, and (B) Kobra Khan, a hybrid of a human and a cobra, included in our sampling.

most recent He-Man animated series showed extremely few female human-animal hybrids, we selected a third science fiction and fantasy setting as a model. Since our aim was to specifically identify female human-animal hybrids, we choose the setting of "She-Ra: Princess of Power". Following the initial success of the "He-Man and the Masters of the Universe" series, Mattel aimed to specifically reach a female audience, and Filmation studios developed the animated series entitled "She-Ra: Princess of Power" encompassing 93 episodes between 1985 and 1987.

The series takes place on the planet Etheria, where the Great Rebellion tries to free the planet from the hideous ruling of the powerful Evil Horde. Odds change in favor of the Rebellion when former Horde captain Adora discovers she is the twin sister of Prince Adam and able to transform into the powerful She-Ra, the female mirror image of He-Man, when wielding a mystical sword akin to the one of He-Man. This series share with "He-Man and the Masters of the Universe" the characteristic of featuring both fantasy and science fiction elements. Despite being a continuation of the initial franchise, the "She-Ra: Princess of Power" series is clearly aimed at a female audience, and therefore, the number of female characters is significantly higher than in most of the other science fiction and fantasy settings. Thus, this setting suited our needs perfectly.

We sampled all the human-animal hybrids featured in the 50 first episodes of the She-Ra: Princess of Power animated series. The sampling approach was exactly the same as the one we described in the previous paragraph (2.2. Corpus 2: He-Man and the Masters of the Universe). For human-animal hybrid characters able to shapeshift (shapeshifting being a relatively common power in the "She-Ra: Princess of Power" setting, e.g. Catra, Hordak, or Imp), only the "main form", defined as the most frequently taken form, was counted.

\section{Statistical Analysis}

For each of the anatomical areas evaluated, percentages were computed for each setting, and within each setting, for each gender. Independent percentages were obtained for morphological and phenotypic alterations. Due to various sizes of the samples, we did not want to assume normality. Therefore, comparisons between phenotypic and anatomical percentages of alterations, and comparisons between male and female sub- 
jects were made using non-parametric Mann-Whitney U test. The non-parametric distribution free Kolmogorov-Smirnov test was used to compare the distributions of patterns of alterations (Eadie et al., 1971; Stephens, 1979). All comparisons of distributions with the Kolmogorov-Smirnov test were performed at the 0.05 level of significance. When applicable, results are presented as mean \pm SEM.

\section{Results}

\section{Star Wars}

Among all the alien characters depicted in the three movies of the prequel Star Wars trilogy, 26 different morphotypes were identified as human-animal hybrids. Surprisingly, all of them were males: while a few Star Wars aliens are female, it was impossible to clearly identify any of them as human-animal hybrids. Only $20.1 \% \pm 3.7 \%$ of the body of the subjects displayed morphological alterations compared to the standard human body form (Figure 3).

When considering the patterns of morphological alterations, i.e., the specific anatomical areas which underwent morphological modification, we uncovered that only a few body areas were favoured as the main targets of body alterations. The pattern of morphological body alteration was clearly not random (significantly different from a random $50 \%$ per area pattern of alteration, Kolmogorov-Smirnov, $p<0.05$ ), but was highly conserved across subjects. Most of the modifications were concentrated in several common areas (e.g., head, hands, and feet), while other areas were almost never altered (e.g., torso, arms). Interestingly, while the hands were often altered, the alterations mostly consisted of a reduction in the number of fingers (from 5 to 4 or 3 ) without other modification of the shape of the hand or its functionality.

In contrast, the proportion of phenotypic alterations observed (clearly non-human skin color, fur, feathers, etc.) was strikingly different. Indeed, the Star Wars human-animal hybrids displayed $85.2 \% \pm 6.7 \%$ of phenotypic body alterations (significantly different from the percentage of morphological alterations, $p<0.001$, Figure 3).

Major deviations from the basic anatomical form of the human body, such as presence of additional limbs, were uncommon. Only $19.23 \%$ of the observed subjects displayed a tail, $11.54 \%$ displayed wings (never resulting in the loss of the hands), $7.69 \%$ a pair of supplementary arms, and none supplementary legs. Major alterations of shape were more common. In our sample, $34.62 \%$ of human-animal hybrids were smaller than standard humans as portrayed in the same setting, $19.23 \%$ were fatter, and only $11.54 \%$ were thinner and $3.85 \%$ taller.

\section{He-Man}

We identified 38 human-animal hybrids in the 39 episodes of the two seasons of the 2002-2004 "He-Man and the Masters of the Universe" animated television series. No gender-ambiguous characters were identified. The overall degree of morphological alterations from human standard anatomy was of $26.6 \% \pm 3.3 \%$ of the body (not significantly different from what was observed for Star Wars, $p=0.122$, (Figure 3). Similarly to what was seen for Star Wars, the phenotypic alterations were considerably higher, affecting $87.3 \% \pm 5 \%$ of the body (significantly different from morphological alterations in He-Man setting, $p<$ 0.001 ; but comparable to the phenotypic alterations in the Star

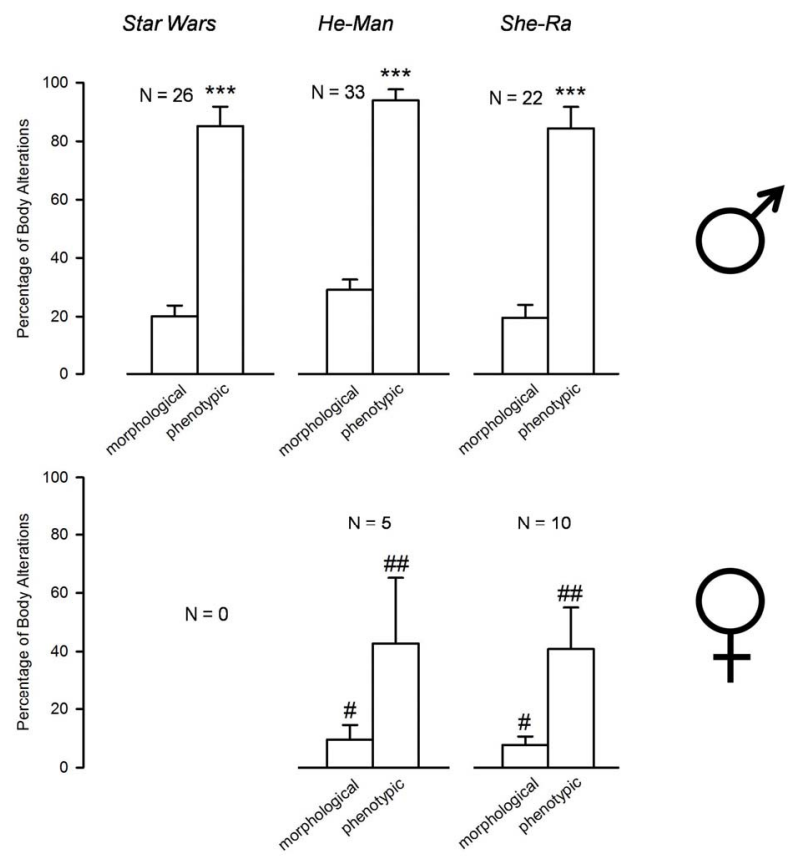

Figure 3.

Percentage of the human body morphologically and phenotypically altered for male and female human-animal hybrids in the three settings observed. This figure shows the percentage of the body altered for male (upper panels) and female (lower panels) human-animal hybrids. For each panel, the left column represent the percentage of morphological alterations, and the right column the percentage of phenotypic alterations. For each setting and gender, the size of the sample (number of subjects) is indicated. ${ }^{* * *}$ denotes a difference between morphology and phenotype of $p<0.001, "$ and ${ }^{\#}$ denotes a difference between males and females of $p<0.05$ and $p<0.01$ respectively.

Wars setting, $p=0.889$, Figure 3).

However, extremely interesting results were observed when considering gender differences. Although not common, a few female human-animal hybrids were identified in the "He-Man" settings. Among the 38 subjects, there were 33 males and 5 females. Striking differences emerged when considering gender as a variable. The percentage of morphological alterations of the 33 males was of $29.2 \% \pm 3.5 \%$. However, the 5 females displayed only $9.6 \% \pm 5 \%$ of their body as morphologically altered. These two proportions were significantly different $(p<$ $0.05)$. The fact that it was possible to statistically assess this difference despite the female group being relatively small confirmed the strength of this effect.

A similar dichotomy was evident when analyzing the phenotypic alterations. While males showed $94.1 \% \pm 3.6 \%$ of phenotypic alteration (not significantly different from the Star Wars setting, $p=0.379$ ), female human-animal hybrids showed only $42.8 \% \pm 22.4 \%$ of phenotypic alteration (significantly different from the He-Man males, $p<0.01$ ).

As observed in the Star Wars setting, additional limbs were relatively uncommon, with only $21.21 \%$ of the males and none of the females displaying supplementary arms (all of them being human-invertebrate hybrids, either human-insects or hu man-arachnids), and no supplementary legs were noted, neither for males nor for females. The occurrence of tails was comparable between males $(21.21 \%)$ and females $(20 \%)$. The case of wings was interesting, as only $18.18 \%$ of the males displayed 
wings, while this proportion reached $80 \%$ among the females we observed.

Again, as in Star Wars, alterations of shape were common. However, the type of shape alterations observed was fully different than in Star Wars. In the He-Man sample, 51.51\% of male human-animal hybrids were bigger (taller and with stronger built), and only $9.09 \%$ were smaller, $6.06 \%$ thinner, and $6.06 \%$ fatter. No shape alterations at all were observed for female human-animal hybrids.

Due to the marked presence of the Snake Men, we were able to identify a sub-group of human-reptile hybrids, consisting of 7 subjects. The case of these human-reptile hybrids was particularly interesting. Indeed, while $25.43 \% \pm 2.19 \%$ of their body showed morphological alterations, $100 \%$ of their body presented phenotypic alterations. Furthermore, the proportion of subjects wielding a tail increased significantly to $57.14 \%$ ( $p$ $<0.05)$. Finally, skull and facial alterations occurred in $100 \%$ of the cases for these hybrids, altogether resulting in a particular and characteristic sub-type.

\section{She-Ra}

Since female human-animal hybrids were not commonly seen in the two previous settings, we specifically considered a "female-enriched" science fiction and fantasy setting, the "She-Ra: Princess of Power" universe. As expected, the universe of She-Ra had an overall higher proportion of female characters, and a significantly higher proportion of female human-animal hybrids. In the first 50 Episodes of the series, we identified 32 human-animal hybrids, including 22 males and 10 females (i.e. a proportion of $24 \%$ of females among human-animal hybrids). No gender-ambiguous characters were identified.

The global morphological alterations observed in the She-Ra setting represented only $15.9 \% \pm 3.3 \%$ of the body, which was not significantly different from what was observed in the Star Wars setting $(p=0.34)$, but significantly different from what was observed in the He-Man setting $(p<0.01)$. This difference can be explained by the high proportion of females within our sample. Indeed, as seen in the He-Man setting, female human-animal hybrids displayed significantly less morphological alterations than males (Figure 3).

When considering only male hybrids, the overall extent of morphological alterations was $19.6 \% \pm 4.4 \%$ of the body, which was still higher than the proportion observed for male hybrids in the He-Man setting $(p<0.05)$, but not significantly different from the proportion of morphological alterations observed in Star Wars $(p=0.983)$. In contrast, only $7.8 \% \pm 2.9 \%$ of the body was morphologically altered for female human-animal hybrids, which was similar to what was observed for the female hybrids from the He-Man setting (not significantly different, $p=0.16$ ), but was significantly different from the morphological alterations in females in the She-Ra setting $(p<0.05)$ and in males in the He-Man $(p<0.001)$ and Star Wars settings $(p<0.05)$.

Concerning the phenotypic alterations, the She-Ra setting replicates what was observed in the two previous settings. The overall proportion of the body displaying phenotypic alterations was $70.8 \% \pm 7.5 \%$, which was not significantly different from either the Star Wars $(p=0.26)$, or the He-Man settings ( $p=$ $0.166)$. Once again, we observed major differences between male and female human-animal hybrids. While males displayed a proportion of $84.5 \% \pm 7.4 \%$ of their body with phenotypic alterations (not significantly different from the males of Star Wars, $p=0.753$, or of He-Man settings, $p=0.716$ ); females displayed much less phenotypic modifications, accounting for only $40.9 \% \pm 14.1 \%$ of their body, which was not significantly different from the proportion of phenotypic alterations observed for He-Man females $(p=0.667)$ but was significantly different from the proportion of phenotypic alterations of She-Ra males $(p<0.01)$, Star Wars males $(p<0.01)$, and He-Man males $(p<$ $0.001)$

The female sampling in the She-Ra setting was large enough to conduct a more detailed analysis of the exact modifications observed. Again, the proportion of female hybrids displaying wings was higher than for male hybrids ( $40 \%$ vs. $22.73 \%$ ). However, apart from wings, female hybrids did not show supplementary limbs $(0 \%$ of supplementary arms, $0 \%$ of supplementary legs, and $10 \%$ of "other" supplementary limbs, in this case due to one human-octopus hybrid displaying two tentacles). Shape alterations were also limited $(20 \%$ of female hybrids being smaller than regular humans, compared to $22.73 \%$ for males; no other shape alterations were evident for female hybrids).

\section{Discussion}

The main conclusion of this study is that human imagination is contrainted by cognitive templates. The process of creation of humanoid creatures in science fiction or fantasy settings is not random, but obeys very strict set of - conscious or unconscious -rules. While the body appearance can be altered, human morphological traits are highly conserved. The patterns of morphological alterations are extremely stereotyped. Finally, female human-animal hybrids retain more human traits than do males (Figure 3).

\section{Patterns of Alterations: Morphology vs. Phenotype}

While phenotypic alterations were extremely common, true anatomical alterations were much more limited. This was first observed in the setting of Star Wars. However, this particular universe presented a few problems: first, despite presenting extremely important diversity (Guitton, 2012b), the Star Wars universe was still only one particular setting; second, despite their famous special effects, the Star Wars movies were still shaped by the limitations inherent of a movie; third, while female aliens were observed, no female hybrids were identified in this setting. Therefore, we decided to choose a second settingHe-Man - to see whether these results could be generalized. This was indeed the case. However, very few females were still observed. Hence, we selected a third setting specifically designed to incorporate a higher proportion of female characters. The results obtained with the She-Ra setting confirmed our conclusions.

Almost all types of animal species were represented in our sampling. Despite a few specific instances related to the settings (see below), modifications were more "cosmetic" than "structural". In other words, the observed modifications affected "aesthetics" rather than "biology". Most modifications consisted solely of theriocephaly (replacing a human head by the head of an animal). Tails, one of the most animalistic and identifiable traits, were largely absent in human-animal hybrids. Thus, we observed more "animal-tainted humans" rather than true animal-human hybrids.

Although being often altered, the hands always kept their 
role as an interface used to manipulate the world. In the vast majority of the cases, the modifications of the hands only consisted of a reduction in the number of fingers. An opposable thumb was almost always present. In the most extreme cases, for instance when the hands were replaced by claws, the capacity to manipulate was nearly intact. The claws of Scorpia (She-Ra universe's woman-scorpion hybrid) allowed her to read a book, and the claws of the crab-men (New He-Man setting) allowed them to tie a rope (Season 1 Episode 22 "The Island") or to hold a sword (Season 1 Episode 25 "The Council of Evil Part 1"). Extremely few hybrids had non-functional hands (for instance, Leech in New He-Man Season 2 Episode 9 "The Power of Grayskull" and in the She-Ra setting, or the spawns of Webstor in New He-Man Season 2 Episode 2 "Web of Evil").

Different sub-types of human-animal hybrids followed more specific patterns. Supplementary limbs were mostly found for human-invertebrate hybrids. In the case of human-bird hybrids, the wings were almost never present instead of hands, but rather in addition to hands (in all the hybrids we observed, only one had wings instead of hands). When the wings were not supplementary limbs, they typically grew out from the forearms, leaving the hands free.

Human-reptile hybrids also followed a specific pattern of alterations, characterized by full phenotypic modifications and an increased probability of displaying a tail. Interestingly, this particular pattern can be observed in other science fiction and fantasy settings, for instance in the Thunder Cats universe with the reptilian "Slithe", the brutish leader of the Plun-Darr mutants (the first villains encountered in the 1985-1989 animated series) or the Lizards (the race of lizard men of the 2011 series), who are extremely comparable in appearance to the He-Man Snake Men. In the world of role-playing games, the "Draconians" and "Troglodytes" of the extremely popular settings of the Forgotten Realms (the Dungeons and Dragons and Advanced Dungeons and Dragons family of role-playing games) are other human-reptile hybrids exemplifying these particularities.

\section{Shape Alterations}

Although the global patterns of anatomical modifications appear to be shared across the different science fiction and fantasy settings we observed, the alterations of global body shape seem to be specific to each particular setting.

For instance, the Star Wars setting seems to favour smaller shapes for human-animal hybrids (a third of the hybrids observed were shorter than a standard human). This particularity extends further than just the human-animal hybrids, and may be seen as a "trademark" of Star Wars humanoid creatures, as observed in the original trilogy with the popular Ewoks (human-bear hybrids inhabiting the forest moon of Endor), or in the Star Wars extended universe with for instance the Mrlssi (human-bird hybrids inhabiting the planet Mrlsst in the Tapani Sector). Similarly, other non human-animal hybrid aliens of the Star Wars setting seem to share this shape alteration, for instance the Jedi Grand Master Yoda, or even droids. Worthy of note, one of the very few characters present in all of the six movies of the two trilogies is the diminutive droid R2-D2.

Symmetrically, the setting of the planet Eternia in the He-Man universe similarly favours a certain type of body shape alteration for the human-animal hybrids (in this case, these hybrids are often bigger and generally more muscular than the average human of the related setting). Once again, this body shape alteration seems to reflect a general trend of the setting, as notably confirmed by the presence of giants (seen for instance in Season 1 Episode 20 "Buzz Off's Pride", or in Season 1 Episode 25 and 26, "Council of Evil, Part 1" and "Council of Evil, Part 2").

This use of alterations of body shape conserved within a given setting appears to be a factor of coherence. Creatures with other shape alterations can of course exist (e.g., Wookiees in Star Wars), but may look "out-of-place" in the given setting (for instance, the Kaminoans seen in Episode II of Star Wars display a combination of body shape alterations relatively unconventional for the Star Wars setting: tall and utterly thin, and "alien-looking" to the Star Wars settings. This unconventional appearance is reinforced by their distinctive architectural and design style, contributing to the general role of an alien species "isolated" from the main galactic influences). The degree of conservation of shape alterations within a particular setting could be seen as a degree of internal coherence of this setting. Not surprisingly, specifically children-targeted settings such as He-Man present a higher conservation of the patterns of shape alterations, allowing for an easier immediate identification and recognition.

\section{Gender Differences}

While human-animal hybrids were a common sight in all of the three science fiction and fantasy settings we studied, female human-animal hybrids were surprisingly extremely rare. Female hybrids were absent or almost absent from the first two settings we studied. Even when looking at a specifically "female-enriched" setting, the proportion of females among human-animal hybrids hardly reached one fourth.

Female hybrids were not only much less common than male hybrids, but also much less altered physically. Indeed, female hybrids displayed far fewer modifications compared to a standard human than males, both in terms of morphological and phenotypic alterations. In addition, alterations of shape were extremely rare, regardless of the particular setting.

Conservation of female traits seems to be critical. Female appearance may bear more constraints for identification; these constraints seem to overpower the creative processes involved in imagination. Similar higher conservation of female features compared to male features has also been evidenced in virtual spaces, through the existence of a compensatory phenomenon between avatar appearance and naming (Guitton, 2010). While there was no difference between male human and non-human avatars, non-human female avatars enriched their names with "female features" to "compensate" for the departure of their appearance from a standard human female by hyper-feminization of their name (Guitton, 2010). From an evolutionary perspective, the "natural" appearance of the human female body could have more meaning than we thought.

\section{Limitations}

While the repetition of the results over three different independent settings seem to indicate that the conclusions could be reasonably generalised, there are still some limitations to this study. First, these three settings may reflect some cultural bias. We choose science fiction and fantasy setting to avoid such bias as much as possible. Second, the role of the expectations of the 


\section{J. GUITTON}

audience should not be underestimated when considering a popular science fiction or fantasy setting. However, it is important to note that this is true too for mythological systems and folkloric tales. Third, one may argue that the observed constraints may be related to commercial issues: most of these visual fictional settings are accompanied by products to be sold as toy lines. Humanoid figurines would be easier to sell due to customer identification process. However, examples taken from toy history of the He-Man franchise seem to contradict this hypothesis. Indeed, humanoid figurines extremely far from standard human template (such as the multi-limbed demon "Modulok") or even purely non-articulated animals (such as the Gringer/Battle Cat toy) were extremely popular and sold very well, despite being actually far more expensive than the standard toys of the line.

\section{Conclusion}

By combining cultural anthropology and anatomical approaches, this study enabled us to unveil some of the fundamental cognitive mechanisms underlying human imagination process. Studying science fiction and fantasy settings allowed us to pin-point some psychological determinants defining the imaginary coherent universes. The process of generation of human-animal hybrids is not random, but follows constraining rules, which are shared across settings. The high degree of conservation of human features, particularly for female hybrids, as well as the presence of repeated patterns of anatomical alterations, suggests the existence of constraining cognitive models of how living organisms should look like. This importance of human morphotype echoes the trends observed in virtual communities in which social organization depend on the degree of proximity of the avatars to human standard appearance (Lortie \& Guitton, 2011). The present study cannot decipher whether the constraining rules observed are conscious or unconscious, or biological or cultural. However, it clearly demonstrates the existence of strong cognitive templates which frame and limit the expression of the capacity of human imagination.

\section{Acknowledgements}

The present work was supported by the Natural Sciences and
Engineering Research Council of Canada (NSERC-grant number 371644). MJG holds a Career Grant from the "Fonds de la Recherche en Santé du Québec” (FRSQ).

\section{REFERENCES}

Black, J. A., \& Green, A. (1992). Gods, demons and symbols of ancient Mesopotamia. Austin, TX: University of Texas Press.

Eadie, W. T., Drijard, D., James, F. E., Roos, M., \& Sadoulet, B. (1971). Statistical methods in experimental physics. Amsterdam: NorthHolland.

Eliade, M. (1965). Rites and symbols of initiation: The mysteries of birth and rebirth. New York: Harper \& Row.

Guitton, M. J. (2010). Cross-modal compensation between name and visual aspect in socially active avatars. Computers in Human Behavior, 26, 1772-1776. doi:10.1016/i.chb.2010.07.004

Guitton, M. J. (2012a). The immersive impact of meta-media in a virtual world. Computers in Human Behavior, 28, 450-455. doi:10.1016/i.chb.2011.10.016

Guitton, M. J. (2012b). Living in the hutt space: Immersive process in the star wars role-play community of second life. Computers in $\mathrm{Hu}$ man Behavior, 28, 1681-1691. doi:10.1016/j.chb.2012.04.006

Harvey, J. S., Watkins, G. M., \& Sherman, R. T. (1984). Emergent burn care. Southern Medical Journal, 77, 204-214. doi:10.1097/00007611-198402000-00019

Howard, R. E. (1929). The shadow kingdom. Weird Tales.

Kasten, K. R., Makley, A. T., \& Kagan, R. J. (2011). Update on the critical care management of severe burns. Journal of Intensive Care Medicine, 26, 223-236. doi:10.1177/0885066610390869

Lomanowska, A. M., \& Guitton, M. J. (2012). Virtually naked: Virtual environment reveals sex-dependent nature of skin disclosure. PLoS ONE, 7, e51921. doi:10.1371/journal.pone.0051921

Lortie, C. L., \& Guitton, M. J. (2011). Social organization in virtual settings depends on proximity to human visual aspect. Computers in Human Behavior, 27, 1258-1261. doi:10.1016/j.chb.2011.01.006

Pinch, G. (2004). Egyptian mythology: A guide to the gods, goddesses, and traditions of ancient Egypt. Oxford: Oxford University Press.

Stephens, M. A. (1979). Test of fit for the logistic distribution based on the empirical distribution function. Biometrika, 66, 591-595.

doi:10.1093/biomet/66.3.591

Wachtel, T. L., Berry, C. C., Wachtel, E. E., \& Frank, H. A. (2000). The inter-rater reliability of estimating the size of burns from various burn area chart drawings. Burns, 26, 156-170. doi:10.1016/S0305-4179(99)00047-9 